PROCEEDINGS OF THE

AMERICAN MATHEMATICAL SOCIETY

Volume 128, Number 1, Pages 27-29

S 0002-9939(99)05101-1

Article electronically published on June 30, 1999

\title{
ON A THEOREM BY SERRE
}

\author{
ARNE LEDET
}

(Communicated by Ronald M. Solomon)

\begin{abstract}
We present a short proof of a theorem by Serre on the trace form of a finite separable field extension.
\end{abstract}

Let $M / K$ be a finite Galois extension in characteristic $\neq 2$, and assume that $M$ is the splitting field over $K$ of an irreducible polynomial $f(X) \in K[X]$ of degree $n$. We embed the Galois group $G=\operatorname{Gal}(M / K)$ transitively into $S_{n}$ by considering the elements of $G$ as permutations of the roots of $f(X)$. From the 'positive' double cover

$$
1 \rightarrow \mu_{2} \rightarrow \widetilde{S}_{n}^{+} \rightarrow S_{n} \rightarrow 1
$$

of $S_{n}$ (i.e., the double cover in which transpositions lift to elements of order 2) we then get an extension

$$
1 \rightarrow \mu_{2} \rightarrow \widetilde{G}^{+} \rightarrow G \rightarrow 1
$$

of $G$ with the cyclic group $\mu_{2}=\{ \pm 1\}$. Let $\gamma^{+} \in H^{2}\left(G, \mu_{2}\right)$ be the characteristic class of $(*)$.

We embed $S_{n}$ into the orthogonal group $O_{n}\left(\bar{K}_{\text {sep }}\right)$ as permutations of the standard basis vectors $\mathbf{e}_{1}, \ldots, \mathbf{e}_{n} \in \bar{K}_{\text {sep }}^{n}$. ( $\bar{K}_{\text {sep }}$ being the separable closure of $K$.) As the pre-image in the Clifford group $C_{n}^{*}\left(\bar{K}_{\mathrm{sep}}\right)$ of a transposition $(i j), i<j$, we can then take the element $x_{i j}=\left(\mathbf{e}_{i}-\mathbf{e}_{j}\right) / \sqrt{2}$. The subgroup of $C_{n}^{*}\left(\bar{K}_{\text {sep }}\right)$ generated by these is exactly the double cover $\widetilde{S}_{n}^{+}$of $S_{n}$, and we get a diagram

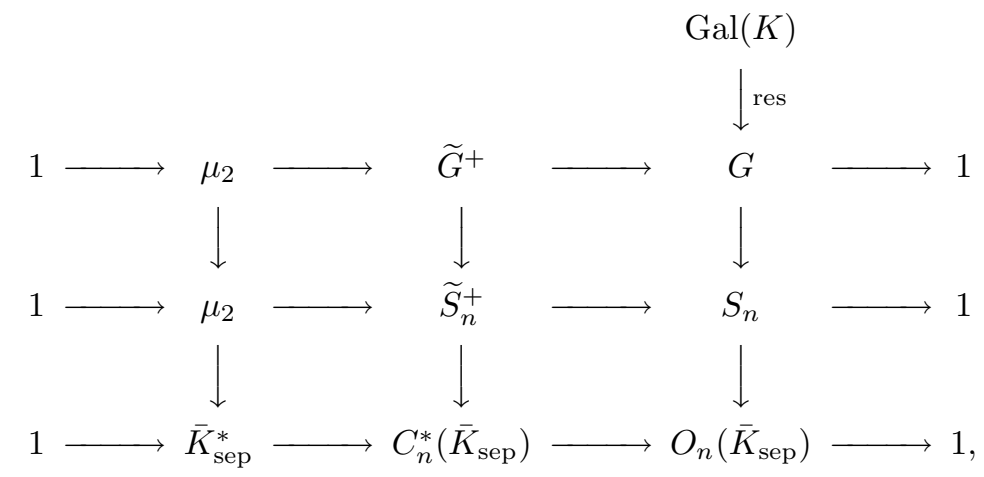

Received by the editors March 7, 1998 .

1991 Mathematics Subject Classification. Primary 12G05.

This work was supported by a Queen's University Advisory Research Committee Postdoctoral Fellowship.

(C)1999 American Mathematical Society 
where $\operatorname{Gal}(K)=\operatorname{Gal}\left(\bar{K}_{\text {sep }} / K\right)$ is the absolute Galois group of $K$. The last row of this diagram is a short-exact sequence of $G$-groups, inducing a connecting map $\delta: H^{1}\left(\operatorname{Gal}(K), O_{n}\left(\bar{K}_{\text {sep }}\right)\right) \rightarrow H^{2}\left(\operatorname{Gal}(K), \bar{K}_{\text {sep }}^{*}\right)$, cf. [Se1], and we know from [Sp] that the image of the crossed homomorphism $e: \operatorname{Gal}(K) \rightarrow O_{n}\left(\bar{K}_{\text {sep }}\right)$ in the last column of the diagram is the Hasse-Witt invariant of the quadratic form obtained from $\langle 1, \ldots, 1\rangle$ by Galois twist with $e$. The Hasse-Witt invariant of a regular quadratic form $q \sim\left\langle a_{1}, \ldots, a_{n}\right\rangle$ is

$$
\mathrm{hw}(q)=\prod_{i<j}\left(a_{i}, a_{j}\right) \in H^{2}\left(\operatorname{Gal}(K), \bar{K}_{\mathrm{sep}}^{*}\right),
$$

where the elements $\left(a_{i}, a_{j}\right) \in H^{2}\left(\operatorname{Gal}(K), \bar{K}_{\text {sep }}^{*}\right)$ are quaternion symbols: For $a, b \in$ $K^{*}$ the quaternion symbol $(a, b)$ is represented by the factor system $(\sigma, \tau) \mapsto$ $(-1)^{\chi_{a}(\sigma) \chi_{b}(\tau)}$, where $\chi_{a}, \chi_{b}: \operatorname{Gal}(K) \rightarrow \mathbb{F}_{2}$ are the homomorphisms with kernels $\operatorname{Gal}(K(\sqrt{a}))$ and $\operatorname{Gal}(K(\sqrt{b}))$, resp.

Now, let $L=K(\theta)$, where $\theta$ is a root of $f(X)$, and let $\theta_{1}=\theta, \theta_{2}, \ldots, \theta_{n} \in M$ be the conjugates. This numbering fixes our embedding of $G$ into $S_{n}$. The Galois twist corresponding to $e$ above is obtained by restricting $\langle 1, \ldots, 1\rangle$ from $\bar{K}_{\text {sep }}^{n}$ to the space of fixed points under the $G$-action ${ }^{\sigma} \mathbf{x}=e_{\sigma}(\sigma \mathbf{x})$. It is easy to see that the fixed points are exactly the points

$$
\left(g\left(\theta_{1}\right), \ldots, g\left(\theta_{n}\right)\right), \quad g(X) \in K[X],
$$

meaning that the twisted quadratic space is $L$ equipped with the trace form $q_{L}$ : $x \mapsto \operatorname{Tr}_{L / K}\left(x^{2}\right)$.

We compute $\delta(e)$ directly as follows: Let $s_{\sigma} \in \widetilde{G}^{+}$be a pre-image of $\sigma \in G$. Then $s_{\text {res } \sigma} \in C_{n}^{*}\left(\bar{K}_{\text {sep }}\right)$ is a pre-image of $e_{\sigma} \in O_{n}\left(\bar{K}_{\text {sep }}\right)$ for $\sigma \in \operatorname{Gal}(K)$, and $\delta(e)$ is given by the factor system

$$
(\sigma, \tau) \mapsto s_{\operatorname{res} \sigma} \sigma s_{\operatorname{res} \tau} s_{\operatorname{res} \sigma \tau}^{-1}=(-1)^{\chi_{2}(\sigma) \chi_{d}(\tau)} s_{\operatorname{res} \sigma} s_{\operatorname{res} \tau} s_{\operatorname{res} \sigma \tau}^{-1}, \quad \sigma, \tau \in \operatorname{Gal}(K)
$$

where $d=d_{L / K}$ is the discriminant of $L / K$, since $\sigma$ operates on $s_{\text {res } \tau}$ through the factor $1 / \sqrt{2}$ contributed by each transposition. Here, $(\sigma, \tau) \mapsto s_{\operatorname{res} \sigma} s_{\operatorname{res} \tau} s_{\operatorname{res} \sigma \tau}^{-1}$ is the inflation of $\gamma^{+}$to $H^{2}\left(\operatorname{Gal}(K), \bar{K}_{\text {sep }}^{*}\right)$, and $(\sigma, \tau) \mapsto(-1)^{\chi_{2}(\sigma) \chi_{d}(\tau)}$ is the quaternion symbol $(2, d)$. Hence, we have

Theorem (Serre, [Se2]). With notation as above,

$$
\inf _{G \rightarrow \operatorname{Gal}(K)}\left(\gamma^{+}\right)=\operatorname{hw}\left(q_{L}\right) \cdot\left(2, d_{L / K}\right) \in H^{2}\left(\operatorname{Gal}(K), \bar{K}_{\text {sep }}^{*}\right) .
$$

If we look instead at the 'negative' double cover

$$
1 \rightarrow \mu_{2} \rightarrow \widetilde{S}_{n}^{-} \rightarrow S_{n} \rightarrow 1
$$

of $S_{n}$, where transpositions lift to elements of order 4 , we get an extension

$$
1 \rightarrow \mu_{2} \rightarrow \widetilde{G}^{-} \rightarrow G \rightarrow 1
$$

of $G$ with $\mu_{2}$. Let $\gamma^{-} \in H^{2}\left(G, \mu_{2}\right)$ be the characteristic class of $(* *)$. The elements $y_{i j}=\left(\mathbf{e}_{i}-\mathbf{e}_{j}\right) / \sqrt{-2} \in C_{n}\left(\bar{K}_{\text {sep }}^{*}\right), i<j$, generate a copy of $\widetilde{S}_{n}^{-}$mapping onto $S_{n} \subseteq O_{n}\left(\bar{K}_{\text {sep }}\right)$, and we can repeat the entire argument above with -2 instead of $2,{ }^{1}$ getting

\footnotetext{
${ }^{1}$ The author would like to thank the referee for suggesting this modified argument.
} 
Theorem. With notation as above,

$$
\begin{gathered}
\inf _{G \rightarrow \operatorname{Gal}(K)}\left(\gamma^{-}\right)=\operatorname{hw}\left(q_{L}\right) \cdot\left(-2, d_{L / K}\right) \in H^{2}\left(\operatorname{Gal}(K), \bar{K}_{\text {sep }}^{*}\right) . \\
\operatorname{REFERENCES}
\end{gathered}
$$

[Se1] J.-P. Serre, Cohomologie Galoisienne, Lecture Notes in Mathematics 5, Springer-Verlag, 1973. MR 53:8030

[Se2] L L'invariant de Witt de la forme $\operatorname{Tr}\left(x^{2}\right)$, Comm. Math. Helv. 59 (1984), 651-676. MR 86k: 11067

[Sp] T. A. Springer, On the Equivalence of Quadratic Forms, Proc. Neder. Acad. Sci. 62 (1959), 241-253. MR 21:7184

Department of Mathematics and Statistics, Queen's University, Kingston, Ontario, Canada K7L 3N6

E-mail address: ledet@mast.queensu.ca 